\title{
Continuous Production of Core-shell Protein Nanoparticles by Antisolvent Precipitation using Dual-channel Microfluidization: Caseinate-coated Zein Nanoparticles
}

\section{Sandra Ebert ${ }^{1,2}$, Charmaine K.W. Koo ${ }^{1}$, Jochen Weiss ${ }^{2}$, and David Julian McClements ${ }^{1 *}$}

\footnotetext{
${ }^{1}$ Biopolymers and Colloids Laboratory, Department of Food Science, University of Massachusetts, Amherst, MA, 01003, USA

${ }^{2}$ Department of Food Biotechnology, University of Hohenheim, Garbenstrasse 25,70599 Stuttgart, Germany
}

Journal: Food Research International

Submitted: November 2016

Running Title: Formation of Core-Shell Biopolymer Nanoparticles 
Antisolvent precipitation is commonly used to fabricate protein nanoparticles using a 3 simple batch method that involves injecting a protein-solvent mixture into an antisolvent. 4 In this study, the potential of producing core-shell protein nanoparticles by antisolvent 5 precipitation using a continuous dual-channel microfluidization method was investigated.

6 The solvent phase (zein in ethanol) and antisolvent phase (casein in water) were made to

7 impinge on each other at high velocity, which generates intense shear, turbulent, and

8 cavitation forces that ensure thorough mixing and breakup of the phases. Relatively

9 small core-shell protein nanoparticles $(d<125 \mathrm{~nm})$ could be produced using this method

10 when the conditions were optimized. The mean particle diameter decreased with

11 increasing antisolvent-to-solvent ratio, increasing homogenization pressure, increasing 12 ethanol content in the solvent phase, and decreasing zein content in the solvent phase.

13 Depending on the processing conditions employed, zein particles in the range of about $14120 \mathrm{~nm}$ to over $1000 \mathrm{~nm}$ could be produced. The operating conditions were further 15 optimized to increase the final zein concentration and decrease the organic solvent 16 content while still obtaining small particles. The surface potential of the core-shell 17 protein nanoparticles went from positive at low $\mathrm{pH}$ to negative at high $\mathrm{pH}$, with a point of 18 zero charge around $\mathrm{pH}$ 5. Electron microscopy indicated that the protein particles formed 19 had a roughly spherical shape. The results suggest that the dual-channel microfluidizer 20 could be used to continuously form protein nanoparticles by antisolvent precipitation. 21 Nevertheless, when the microfluidization method was compared with the simple batch 22 method the size of the particles produced under similar conditions were fairly similar.

23 Keywords: microfluidizer; antisolvent precipitation; zein; caseinate; nanoparticles; core24 shell; delivery systems 


\section{Introduction}

Protein nanoparticles and microparticles may be utilized as natural functional

28 ingredients in numerous applications in the food industry, including as colloidal delivery 29 systems, stabilizers, lightening agents, and texture modifiers [1-4]. The ability of protein 30 particles to provide these desirable functional attributes depends on particle properties, 31 such as composition, size, morphology, charge, and polarity [5]. Consequently, there is 32 considerable interest in developing processing methods to fabricate protein particles with 33 well-defined physicochemical and structural properties [6]. A particularly promising 34 method for producing protein particles is antisolvent precipitation [7-10]. Traditionally, 35 this method is carried out using a simple batch process where a protein/solvent mixture is 36 injected into an antisolvent, which leads to the spontaneous formation of small protein 37 particles. These particles form due to self-association of the protein molecules when the 38 solvent quality changes. For hydrophobic proteins, an organic liquid (such as ethanol) is used as the solvent phase, and water is used as the antisolvent phase [11]. Conversely,

40 for hydrophilic proteins, water is used as the solvent phase, and an organic liquid is used 41 as the antisolvent phase $[12,13]$.

42 In the current study, a hydrophobic protein (zein) was used to form the core of the 43 protein-nanoparticles, whereas as an amphiphilic protein (caseinate) was used to form the 44 shell. This combination was employed because it has previously been shown that 45 relatively small stable core-shell protein nanoparticles can be formed with the antisolvent 46 precipitation method [10, 14, 15]. In this case, a mixture of zein and ethanol are injected 47 into an aqueous buffer solution consisting of caseinate molecules dissolved in water (at a $48 \mathrm{pH}$ away from the isoelectric point). After injection, the zein molecules associate with 49 each other when the ethanol diffuses into the surrounding aqueous phase, and the local 50 ethanol concentration surrounding the zein molecules drops below the solubilization limit 51 thereby causing protein precipitation [16]. The caseinate is needed in the aqueous

52 solutions to adsorb to the surfaces of the zein particles and cover any exposed non-polar 53 patches. In the absence of this emulsifier, the bare zein nanoparticles have a strong 54 tendency to aggregate due to a strong hydrophobic attraction between the exposed non55 polar patches. The adsorption of the caseinate molecules reduces the surface 
56 hydrophobicity, and can increase the steric and electrostatic repulsion between the

57 particles, thereby improving their aggregation stability.

58 As mentioned earlier, protein nanoparticles are normally formed by the antisolvent

59 precipitation using a simple batch approach that involves injecting a protein-solvent

60 mixture into an antisolvent. Recently, it was shown that protein nanoparticles can also be

61 produced by antisolvent precipitation using an impinging jet approach [17]. The authors

62 constructed a mechanical device that consisted of two syringes, one containing the

63 protein-solvent and the other containing the antisolvent. The syringe outlets were

64 connected to a specially designed series of channels that made the two fluid streams

65 impinge upon each other at high velocity. This procedure led to the formation of small

66 protein particles, which was mainly attributed to the intense mixing forces generated

67 when the two liquids collided with each other. In the present study, we investigated the

68 possibility of using a commercially available dual-channel microfluidizer (PureNano ${ }^{\circledR}$,

69 Microfluidics, Newton, MA) to continuously form protein nanoparticles using the

70 antisolvent precipitation method. The principle behind the microfluidization method is

71 similar to the impinging jet approach, but the former is a continuous method that could be

72 used at an industrial scale. As part of this study, we examined the impact of various

73 processing parameters on particle properties so that we could form small protein particles

74 with low amounts of organic solvent. We also compared the properties of the protein

75 particles produced using the microfluidization method with those produced using the

76 simple batch injection method to compare their potential advantages and disadvantages.

\section{2. Materials and methods}

\section{2.1. Materials}

79 Powdered zein ( $\geq 95 \%$ ) was purchased from Sigma Aldrich Corp. (St. Louis MO, 80 USA). Sodium caseinate and ethanol (100\% proof) were purchased from Thermo Fisher 81 Scientific (Waltham, MA, USA). Other chemicals, reagents, and solvents were purchased

82 from Sigma-Aldrich or Thermo Fisher Scientific (Waltham, MA, USA) and were of

83 analytical grade, unless otherwise stated. 


\section{2.1. Solution preparation}

85 For the solvent phase, hydrophobic zein from maize was dissolved in a mixture of 86 ethanol and $10 \mathrm{mM}$ citrate phosphate buffer $(\mathrm{pH} 7)$. The solution was stirred for $2 \mathrm{~h}$ at

87 room temperature to ensure complete hydration of the protein. Solutions were

88 subsequently vacuum filtered with a Whatman Filter (Nr. 42). 1 \% sodium caseinate was

89 dissolved in $10 \mathrm{mM}$ citrate phosphate buffer ( $\mathrm{pH}$ 7.0) to prepare the antisolvent phase.

\section{2.2. Nanoparticle Preparation}

91 Continuous Method: The fabrication of nanoparticles was carried out using a high 92 pressure dual-channel microfluidizer (Microfluidics PureNano, Newton, MA, USA) with

93 Y- and Z-type interaction chambers. The impact of operating pressure (5 to $19 \mathrm{kpsi}$ ),

94 solvent-to-antisolvent ratio (1:10 to $1: 1)$, zein concentration (1 to $10 \% \mathrm{w} / \mathrm{v}$ ), and ethanol

95 concentration $(60-85 \% \mathrm{v} / \mathrm{v})$ was varied. Based on preliminary experiments, the aqueous

96 phase composition was kept constant, consisting of $1 \%(\mathrm{w} / \mathrm{v})$ sodium caseinate dissolved

97 in citrate phosphate buffer solution (10 mM, pH 7.0).

98 The solvent and antisolvent were poured into the two input hoppers of the

99 microfluidizer, and then forced into the mixing chamber under pressure at variable

100 relative flow rates (to alter the solvent-to-antisolvent ratio). Antisolvent precipitation was

101 achieved using a single pass of the samples through the device. After particle formation,

102 ethanol was removed using a vacuum rotary evaporator at $40^{\circ} \mathrm{C}$ (Buchi RE 111, Flawil,

103 Switzerland).

104 Batch Method: A simple batch injection method was also used to prepare protein

105 particles by antisolvent precipitation under similar conditions as the dual-channel

106 microfluidizer (with the exception of pressure variation). In this case, the solvent was

107 poured into the antisolvent at a stirring speed of $600 \mathrm{rpm}$ and then the sample was stirred

108 for a further $5 \mathrm{~min}$. Ethanol was removed using the rotary evaporation method mentioned 109 earlier.

110 Sodium azide $(0.02 \% \mathrm{w} / \mathrm{v})$ was added to all samples after particle formation to 111 prevent any microbial growth during storage. 


\section{2.3. Nanoparticle Characterization}

113 Particle size and charge: The size and charge of the protein particles was measured

114 using a combined dynamic light scattering / particle electrophoresis instrument (Nano ZS,

115 Malvern Instruments, Malvern, UK). Samples were diluted in $10 \mathrm{mM}$ citrate phosphate

116 buffer solutions at the appropriate $\mathrm{pH}$ prior to characterization to prevent multiple

117 scattering effects. The instrument reports the mean particle diameter (Z-average size),

118 polydispersity index (PDI), and $\zeta$-potential of the protein particles. Samples were

119 equilibrated at $25^{\circ} \mathrm{C}$ prior to analysis.

$120 \quad$ pH stability: The $\zeta$-potential versus $\mathrm{pH}$ profiles of zein nanoparticles, caseinate/zein-

121 coated nanoparticles, and caseinate solutions $(1 \% \mathrm{w} / \mathrm{v})$ were measured. All samples were

122 prepared at $\mathrm{pH} 7$ and adjusted to $\mathrm{pH}$ values ranging from 2 to 9 with phosphoric acid and

123 sodium hydroxide, respectively.

124 Particle morphology: Aqueous protein particle suspensions were freeze-dried and

125 then the resulting powder was visualized using SEM to provide information about

126 particle morphology. A thin layer of the powder was immobilized on double-sided carbon

127 tape. The samples were subsequently sputter coated with 3-6 nm of platinum. The

128 samples were then visualized on a Magellan 400 FESEM (FEI, Hillsboro. OR, USA) at 5

$129 \mathrm{kV}$ and $13 \mathrm{pA}$.

\section{$130 \quad$ 2.4. Statistical analysis}

131 All measurements were performed at least twice on duplicate freshly prepared

132 samples. Means and standard deviations were calculated from these four measurements

133 using Excel (Microsoft, Redmond, VA, USA). One-way analysis of variance was carried

134 out with IBM SPSS Statistics (IBM Corp, Armonk, NY, USA). Differences among pairs

135 of means were compared using a Tukey-test and a significance level of 0.05 . 
147 solvent. 148 3.1. Influence of operating pressure

\section{Results and Discussion}

Suspensions of protein particles, hereafter referred to as hydrosols, were prepared by mixing solvent and antisolvent, using either the dual-channel microfluidizer or the simple batch approach. A solvent phase containing $3 \%$ zein (w/v) dissolved in $80 \%$ ethanol $(\mathrm{v} / \mathrm{v})$ solution, and a solvent-to-antisolvent ratio of 1-to-5 were used as initial parameters for particle formation based on previous studies (Davidov-Pardo, Joye, and McClements 2015; Zhong and Jin 2009). Preliminary experiments showed that no protein purification of the solvent phase, in form of centrifugation, was needed prior to particle preparation by antisolvent precipitation, as there was no significant difference ( $p>0.05)$ among these samples concerning particle size and PSD. The $\zeta$-potential was approximately $-30 \mathrm{mV}$ for all samples, except for samples prepared by varying the zein concentration in the

Increasing the operating pressure of the microfluidizer should generate more intense disruptive forces when the protein-solvent and antisolvent mixtures collide, which would be expected to influence the particle size. The influence of the operating pressure on the particle size was therefore investigated, keeping the other system parameters constant (Figure 2). At all operating pressures, the particle size distribution was monomodal, the polydispersity index was relatively small (PDI $<0.21$ ), and the mean particle diameter was $<200 \mathrm{~nm}$, indicating that even low pressures were suitable for producing core-shell protein nanoparticles. Nevertheless, the mean particle diameter did decrease with increasing operating pressure from 5 to $15 \mathrm{kpsi}$, and then remained fairly constant (Fig. 2a). Statistical analysis indicated that only the mean diameters of the protein particles produced at 5 and $7 \mathrm{kpsi}$ were significantly different $(\mathrm{p}>0.05)$ from those produced at 9 to $19 \mathrm{kpsi}$. Thus, it may be beneficial to use higher pressures in applications where smaller particles are required. Presumably higher pressures lead to better mixing of the solvent and antisolvent phases, and may help break up any aggregated protein particles that may be formed. Protein particle aggregation may occur if the bare zein nanoparticles collide with each other before they have time to be coated by caseinate molecules. Nevertheless, overall these experiments suggest that the operating pressure of 
166 the microfluidizer does not have a major impact on the size of the protein particles

167 produced. This is consistent with a previous study that reported that there was a decrease

168 in protein particle size when the velocity that the solvent and antisolvent were made to

169 impinge on each other increased [17].

\section{$170 \quad 3.2$. Influence of solvent to antisolvent ratio}

171 An increase in the concentration of protein nanoparticles using the microfluidization 172 methods would be beneficial for the industrial application of this technology. One means

173 of increasing the nanoparticle level in the final systems is to increase the solvent-to-

174 antisolvent ratio utilized. The impact of varying the solvent-to-antisolvent mixing ratio

175 was therefore examined while all the other parameters were kept constant: $3 \%$ zein (w/v)

176 in $80 \%$ ethanol solution (v/v) for the solvent phase, $1 \%$ sodium caseinate $(\mathrm{w} / \mathrm{v})$ in the

177 water phase; and an operating pressure of $13 \mathrm{kpsi}$.

178 There was little impact of the mixing ratio on the particle size distribution or mean

179 particle diameter from 1:10 to 1:4 solvent-to-antisolvent, with the mean particle diameter 180 remaining appreciably below $200 \mathrm{~nm}$ (Figure 3). However, there was an appreciable 181 increase in the particle size when the solvent-to-antisolvent ratio was increased further, 182 with very large particles (> $2000 \mathrm{~nm}$ ) being formed at a 1:1 ratio. Interestingly, all of the 183 systems had a monomodal particle size distribution, regardless of the mixing ratio used.

184 The reason that small particles were produced at low mixing ratios may be attributed to 185 the impact of solvent composition on saturation, nucleation, and growth processes [7]. 186 When there is a large amount of antisolvent (water) present, the ethanol rapidly diffuses 187 out of the protein-solvent particles and the critical saturation concentration where protein 188 precipitation occurs is reached rapidly. Consequently, relatively small particles are 189 formed under these conditions. Conversely, when there is a much lower amount of 190 antisolvent present, the critical saturation concentration is reached more slowly.

191 Consequently, the particles formed may have a more diffuse and irregular structure that is 192 more prone to aggregation. Moreover, there would have been less caseinate present at 193 the higher solvent-to-antisolvent ratios, which means that the protein particles could have 194 had a greater tendency to aggregate due to hydrophobic attraction.

195 These results indicate that there is a range of solvent-to-antisolvent ratios that can be 196 utilized to produce small protein nanoparticles using the microfluidization method. 


\subsection{Influence of ethanol content}

From an industrial viewpoint, it is advantageous to minimize the amount of organic

200 solvent required to fabricate the protein nanoparticles from an economical and

201 environmental perspective [18]. To investigate the minimal needed amount of organic

202 solvent required, the ethanol content in the solvent phase was varied from $60-85 \%(\mathrm{v} / \mathrm{v})$,

203 with the remainder being citrate phosphate buffer. This range was determined to be the

204 solubility limit of the hydrophobic biopolymer zein in the ethanol-buffer mixture. The

205 protein was found to precipitate from the solvent phase when the ethanol level was below

$20660 \%$ or above $85 \%$, which is in accordance to previous studies on similar systems [11].

207 For all ethanol levels used, the mean diameter of the protein particles produced was

208 relatively small $(<180 \mathrm{~nm})$ and the particle size distribution was monomodal (Figures 4

209 and 4b). Nevertheless, there was a moderate decrease in mean particle diameter with

210 increasing ethanol concentration, from around 175 to $158 \mathrm{~nm}$. Interestingly, the opposite

211 effect was reported in a previous study where antisolvent precipitation was carried out

212 using a jet impingement method [17]. However, again the mean particle diameter did not

213 depend strongly on ethanol concentration in that study either, varying from around 120 to

$214160 \mathrm{~nm}$. Overall, these results suggest that ethanol level did not have a major influence,

215 and that it would be possible to form relatively small particles over a range of ethanol 216 levels.

\section{3.4. Influence of zein concentration}

218 An alternative approach to increase the total concentration of protein nanoparticles in 219 the final hydrosol formed after antisolvent precipitation is to increase the zein level in the 220 initial protein-solvent solution $[11,15]$. The impact of varying the zein concentration in 221 the solvent phase from 1 to $10 \%(\mathrm{w} / \mathrm{v})$, while keeping all other parameters fixed was 222 therefore investigated.

223 There was an increase in mean particle diameter from around 141 to $556 \mathrm{~nm}$ with 224 increasing zein concentration in the solvent phase (Figure 5a). From about 1 to $4 \%$ 225 protein the mean particle diameter was relatively small $(<200 \mathrm{~nm})$ and the particle size 226 distribution was monomodal (Figure 5b), but at higher values that was a steep increase in 
227 particle diameter, and the polydispersity increased (Figure 5b). This effect may be 228 attributed to a number of factors. First, there may have been insufficient caseinate 229 present in the antisolvent (water) phase to completely cover all of the zein particles 230 formed, thereby leading to bridging flocculation [19]. Second, the high initial protein

231 level in the solvent phase will have led to a more concentrated suspension of protein 232 particles being formed, thereby increasing the chance of particle aggregation due to the 233 increase in the particle collision frequency in more concentrated systems [7]. Third, the

234 increase in the viscosity of the protein-solvent solution may have hindered ethanol

235 diffusion out of the droplets and/or inhibited the intense mixing of the solvent and 236 antisolvent.

237 Measurements of the surface potential of the protein particles was carried out to 238 provide additional insights into the origin of this effect. The $\zeta$-potential of the protein 239 particles became more negative with increasing zein concentration, changing from 240 around -27 to $-34 \mathrm{mV}$ as the protein level was increased from 1 to $10 \%$ (data not shown). 241 At neutral $\mathrm{pH}$, the $\zeta$-potential of the caseinate molecules was less negative than that of the 242 zein nanoparticles (see later). This suggests that the zein particles may not have been 243 fully coated by sodium caseinate at high zein levels, which could have led to some 244 particle aggregation. The particles may have aggregated due to hydrophobic attraction 245 between exposed non-polar groups on the zein particle surfaces, or due to bridging 246 flocculation caused by a single caseinate molecule adsorbing to the surface of multiple 247 zein particles. In future studies, it would therefore be interesting to examine the influence 248 of caseinate concentration on the formation of stable core-shell nanoparticles.

\section{3.5. Optimized parameters for antisolvent precipitation}

250 Based on the results of the previous experiments, the following optimized parameters 251 were selected for creating core-shell protein nanoparticles with small mean particle 252 diameters and monomodal particle size distributions: solvent phase: $4 \%$ zein (w/v) and $25380 \%$ ethanol (v/v) in buffer solution; antisolvent phase: $1 \%$ sodium caseinate in buffer 254 solution; solvent-to-antisolvent mixing ratio $=1$ to 5 ; and, operating pressure $=13 \mathrm{kpsi}$. 


\subsection{Electrical characteristics of core-shell protein particles}

256

257

258

259

260

261

262

263

264

265

266

267

268

269

270

271

272

273

274

275

276

277

278

279

280

281

282

283

284

285

A number of important functional attributes of protein particles depend on their electrical characteristics, such as their aggregation stability, tendency to adsorb to charged surfaces, and interaction with other charged species. The electrical properties of proteins are $\mathrm{pH}$-dependent, which effects their ability to exhibit their desired functional attributes in different types of food products or within the gastrointestinal tract where the $\mathrm{pH}$ can change considerable. For this reason, the $\zeta$-potential versus $\mathrm{pH}$ profile of the caseinate-coated zein protein nanoparticles was compared to bare zein nanoparticles and caseinate molecules (Figure 6).

The surface potential of all the samples ranged from highly positive to highly negative as the $\mathrm{pH}$ was increased from highly acidic $(\mathrm{pH} 2)$ to highly basic (pH 9) (Fig. 6a. This behaviour is typical of proteins and can be attributed to the presence of both amine groups and carboxylic groups on the polypeptide chains. In general, a protein is negatively charged above its isoelectric point (pI), but positively charged below it. The caseinate had a lower pI (4.5) than the bare zein nanoparticles (5.9). Extensive protein aggregation and sedimentation was observed in these samples because the relatively weak electrostatic repulsion between them was insufficient to overcome the attractive interactions (such as van der Waals and hydrophobic attraction). As expected, the coreshell protein nanoparticles had a pI (5) between that of the bare zein nanoparticles and casein molecules. In addition, the $\zeta$-potential of the core-shell nanoparticles was appreciably different from that of the bare nanoparticles at most $\mathrm{pH}$ values, which suggests that the caseinate molecules were absorbed. The interaction of zein and sodium caseinate may be attributed to hydrophobic interactions, or interactions between cationic groups of one protein and anionic groups of the other. At very high $(\mathrm{pH} \mathrm{9)}$ and very low $(\mathrm{pH} 2) \mathrm{pH}$-values the core-shell nanoparticles had surface potentials about the same magnitude as bare zein nanoparticles, which suggests that the caseinate may not have adsorbed under these conditions. Presumably, there was a large electrostatic repulsion between the two proteins under these conditions, which was large enough to overcome the hydrophobic attraction.

The particle size measurements and visual observations indicated that there was extensive aggregation in all three systems at $\mathrm{pH} 5$ (Fig. 6b), which is close to the 
286 isoelectric point of the proteins (Fig. 6a). The caseinate solutions appeared optically

287 clear at $\mathrm{pH}$ 2, 3, 7 and 9, which suggests that the proteins were dissolved under these

288 conditions. The extensive aggregation observed at $\mathrm{pH} 5$ in all the samples can be

289 attributed to the reduction in the electrostatic repulsion between the protein molecules

290 and particles.

\section{3.7. Comparison of continuous and batch antisolvent precipitation methods}

292 In this section, the sizes of the particles produced using the continuous dual-channel

293 microfluidization method were compared with those produced using the traditional batch

294 antisolvent precipitation method. Initially, we hypothesized that much smaller particle

295 sizes would be produced using the microfluidization method due to the intense mixing

296 conditions generated when the solvent and antisolvent phases were made to collide

297 together. However, in practice it was found that in most cases the size of the protein

298 particles produced using the batch method were very similar to those produced using the

299 microfluidization method using similar system conditions (e.g., similar zein

300 concentration, ethanol concentration, and solvent-to-antisolvent mixing ratio). This

301 effect is illustrated in Figure 7, where the mean diameters of the particles produced using

302 the batch method are plotted against those produced using the microfluidizer method

303 under similar conditions. Under conditions where relatively small protein particles were

304 formed $(\mathrm{d}<500 \mathrm{~nm})$ there was a strong linear correlation between the two different

305 antisolvent precipitation methods $\left(r^{2}=0.89\right.$, slope $\left.=0.90\right)$. Conversely, under conditions

306 where very large particles tend to be produced (such as high zein levels) the

307 microfluidizer generates considerably smaller particles than the batch method,

308 presumably because the intense disruptive forces in the mechanical device can partially

309 breakup large aggregates. These results suggest that the mechanism of nanoparticle

310 formation does not depend strongly on the mixing conditions used. Overall, it seems that

311 the microfluidizer is not able to produce smaller particles, but that its main advantage is

312 that it is a rapid continuous process that could produce protein particles on a larger scale

313 than the simple batch process. In addition, it was observed that the reproducibility of the

314 particle size produced using a given set of conditions was better (smaller standard

315 deviation from batch-to-batch) for the microfluidizer (Mean Error $=4.8 \%$ ) than for the 
316 batch method (Mean Error $=7.3 \%$ ), which may also be important for some commercial 317 applications.

\subsection{Microstructure of nanoparticles}

320 Finally, the microstructure of the protein particles produced using the batch and 321 continuous (microfluidizer) approach was characterized using scanning electron 322 microscopy (SEM). In general, both samples contained a large number of roughly 323 spherical particles (Figure 8). The roughness of the protein particle surfaces may have 324 been an artifact introduced by the freeze-drying and electron microscopy preparation 325 procedure. Interestingly, an occasional SEM image (about 1 in 10) of the samples 326 prepared using the batch method indicated that they contained some large particles, 327 whereas those of the samples produced using the microfluidizer method always contained 328 small particles (data not shown). The presence of these large particles was not detected 329 by the dynamic light scattering method used, since the mean particle diameters were 330 fairly similar for both the batch and microfluidizer methods (Figure 7). This may have 331 been because these large particles rapidly sediment to the bottom of the measurement 332 cells in the dynamic light scattering instrument and are therefore not detected. These 333 results suggest that there may be an advantage in using the microfluidization method if a 334 highly uniform particle suspension is required. It was not possible to verify the expected 335 core-shell structure of the protein nanoparticles using the SEM method because it only 336 gives information about the surface topology. In future studies, it would be useful to 337 utilize TEM for this purpose.

\section{Conclusions}

This study has shown that protein nanoparticles can be successfully produced by 341 antisolvent precipitation using a dual-channel microfluidizer. The main advantage of the 342 microfluidization method over conventional batch methods for this purpose is that it can 343 rapidly and continuously produce particles, which may make it more suitable for large344 scale production purposes. A number of process variables were examined to determine 345 the magnitude of their impact on the size of the protein particles produced by the 
346 microfluidizer. The particle size could be reduced by increasing homogenization

347 pressure, reducing the solvent-to-antisolvent mixing ratio, increasing the ethanol content

348 in the solvent phase, or increasing the zein level in the solvent phase. Under optimized

349 conditions a suspension of relatively small $(\mathrm{d}<130 \mathrm{~nm})$ caseinate-coated zein particles

350 with a monomodal particle size distribution could be produced. Interestingly, the size of

351 the particles produced by the microfluidizer method were similar to those produced using

352 the conventional batch antisolvent precipitation method under conditions where small

353 particles were generated. This observation suggests that the highly intense mixing

354 conditions produced within the microfluidizer did not play a critical role in the formation

355 of small protein nanoparticles by antisolvent precipitation. Nevertheless, they were

356 capable of partially breaking up aggregates under conditions where large particles are

357 formed.

358 In summary, the dual channel microfluidization method is capable of rapidly and

359 continuously producing core-shell protein nanoparticles using the antisolvent

360 precipitation approach. These features may make it suitable for the large-scale

361 commercial production of protein nanoparticles that could be used as delivery systems,

362 stabilizers, lightening agents, or texture modifiers. The same approach may also be

363 useful for producing other types of food-grade nanoparticles. For example,

364 nanoemulsions can be produced by passing oil through one channel and water through

365 the other, whereas nutraceutical nanocrystals can be formed by passing a hydrophobic

366 nutraceutical dissolved in an organic solvent through one channel and water through the

367 other.

368

\section{Acknowledgements}

370 Sandra Ebert gratefully acknowledges financial support from the PROMOS

371 scholarship committee during the project. We thank Long Bai for his advice and help in

372 operating the microfluidizer. This material was also partly based upon a grant from the

373 Cooperative State Research, Extension, Education Service, USDA, Massachusetts

374 Agricultural Experiment Station (MAS00491) 
375

376

377

378

379

380

381

382

383

384

385

386

387

388

389

390

391

392

393

394

395

396

397

398

399

400

401

402

403

404

405

\section{References}

Augustin, M. A. 2003. "The Role of Microencapsulation in the Development of Functional Dairy Foods." ResearchGate 58 (2): 156-60.

Bai, Long, Siqi Huan, Jiyou Gu, and David Julian McClements. 2016. "Fabrication of Oil-in-Water Nanoemulsions by Dual-Channel Microfluidization Using Natural Emulsifiers: Saponins, Phospholipids, Proteins, and Polysaccharides.” Food Hydrocolloids 61 (December): 703-11. doi:10.1016/j.foodhyd.2016.06.035.

Bai, Long, and David Julian McClements. 2016. "Development of Microfluidization Methods for Efficient Production of Concentrated Nanoemulsions: Comparison of Single- and Dual-Channel Microfluidizers." Journal of Colloid and Interface Science 466 (March): 206-12. doi:10.1016/j.jcis.2015.12.039.

Davidov-Pardo, Gabriel, Iris J. Joye, and David Julian McClements. 2015. "Encapsulation of Resveratrol in Biopolymer Particles Produced Using Liquid Antisolvent Precipitation. Part 1: Preparation and Characterization." Food Hydrocolloids 45 (March): 309-16. doi:10.1016/j.foodhyd.2014.11.023.

Grodowska, Katarzyna, and Andrzej Parczewski. 2010. “Organic Solvents in the Pharmaceutical Industry." Acta Poloniae Pharmaceutica 67 (1): 3-12.

Guzey, Demet, and D. Julian McClements. 2006. "Formation, Stability and Properties of Multilayer Emulsions for Application in the Food Industry." Advances in Colloid and Interface Science, In Honor of Professor Nissim Garti’s 60th Birthday, 128130 (December): 227-48. doi:10.1016/j.cis.2006.11.021.

Hinrichs, J. 2001. "Incorporation of Whey Proteins in Cheese.” International Dairy Journal, Cheese Ripening and Technology, 11 (4-7): 495-503. doi:10.1016/S0958-6946(01)00071-1.

Joye, Iris Julie, and David Julian McClements. 2013. "Production of Nanoparticles by Anti-Solvent Precipitation for Use in Food Systems." Trends in Food Science \& Technology 34 (2): 109-23. doi:10.1016/j.tifs.2013.10.002.

Kryder, David, Stanley Kowalski, and Anatole Krattiger. 2000. "The Intellectual and Technical Property Components of pro-Vitamin A Rice (GoldenRiceTM): A Preliminary Freedom-To-Operate Review.” Legal Scholarship, January. http://scholars.unh.edu/law_facpub/167. 
406 Luo, Yangchao, Zi Teng, Thomas T. Y. Wang, and Qin Wang. 2013. "Cellular Uptake 407 and Transport of Zein Nanoparticles: Effects of Sodium Caseinate." Journal of Agricultural and Food Chemistry 61 (31): 7621-29. doi:10.1021/jf402198r.

1. Jones, O.G. and D.J. McClements, Functional biopolymer particles: design, fabrication, and applications. Comprehensive Reviews in Food Science and Food Safety, 2010. 9(4): p. 374-397. 
2. Joye, I.J. and D.J. McClements, Biopolymer-based nanoparticles and microparticles: Fabrication, characterization, and application. Current Opinion in Colloid \& Interface Science, 2014.

3. Xiao, J., Y.Q. Li, and Q.R. Huang, Recent advances on food-grade particles stabilized Pickering emulsions: Fabrication, characterization and research trends. Trends in Food Science \& Technology, 2016. 55: p. 48-60.

4. Chen, L.Y., G.E. Remondetto, and M. Subirade, Food protein-based materials as nutraceutical delivery systems. Trends in Food Science \& Technology, 2006. 17(5): p. 272-283.

5. McClements, D.J., Nanoparticle- and Microparticle-based Delivery Systems. First ed. 2015, Boca Raton, FL: CRC Press.

6. Matalanis, A., O.G. Jones, and D.J. McClements, Structured biopolymer-based delivery systems for encapsulation, protection, and release of lipophilic compounds. Food Hydrocolloids, 2011. 25(8): p. 1865-1880.

7. Joye, I.J. and D.J. McClements, Production of nanoparticles by anti-solvent precipitation for use in food systems. Trends in Food Science \& Technology, 2013. 34(2): p. 109-123.

8. Thorat, A.A. and S.V. Dalvi, Liquid antisolvent precipitation and stabilization of nanoparticles of poorly water soluble drugs in aqueous suspensions: Recent developments and future perspective. Chemical Engineering Journal, 2012. 181: p. 1-34.

9. Patel, A., et al., Synthesis and characterisation of zein-curcumin colloidal particles. Soft Matter, 2010. 6(24): p. 6192-6199.

10. Patel, A.R., E.C.M. Bouwens, and K.P. Velikov, Sodium Caseinate Stabilized Zein Colloidal Particles. Journal of Agricultural and Food Chemistry, 2010. 58(23): p. 12497-12503.

11. Zhong, Q.X. and M.F. Jin, Zein nanoparticles produced by liquid-liquid dispersion. Food Hydrocolloids, 2009. 23(8): p. 2380-2387.

12. Gulseren, I., Y. Fang, and M. Corredig, Whey protein nanoparticles prepared with desolvation with ethanol: Characterization, thermal stability and interfacial behavior. Food Hydrocolloids, 2012. 29(2): p. 258-264.

13. Gulseren, I., Y. Fang, and M. Corredig, Zinc incorporation capacity of whey protein nanoparticles prepared with desolvation with ethanol. Food Chemistry, 2012. 135(2): p. 770-774.

14. Li, K.K., et al., Preparation of water-soluble antimicrobial zein nanoparticles by a modified antisolvent approach and their characterization. Journal of Food Engineering, 2013. 119(2): p. 343-352.

15. Davidov-Pardo, G., I.J. Joye, and D.J. McClements, Encapsulation of resveratrol in biopolymer particles produced using liquid antisolvent precipitation. Part 1: Preparation and characterization. Food Hydrocolloids, 2015. 45: p. 309-316.

16. Zhong, Q.X., et al., Application of supercritical anti-solvent technologies for the synthesis of delivery systems of bioactive food components. Food Biophysics, 2008. 3(2): p. 186-190.

17. Li, K.K., et al., Continuous preparation of zein colloidal particles by Flash NanoPrecipitation (FNP). Journal of Food Engineering, 2014. 127: p. 103-110. 
481 18. Grodowska, K. and A. Parczewski, Organic solvents in the pharmaceutical industry. 482 Acta Poloniae Pharmaceutica, 2010. 67(1): p. 3-12.

483 19. Guzey, D. and D.J. McClements, Formation, stability and properties of multilayer 484 emulsions for application in the food industry. Advances in Colloid and Interface $485 \quad$ Science, 2006. 128: p. 227-248.

486 


\section{Inlet reservoir 1}

- Zein in Ethanol

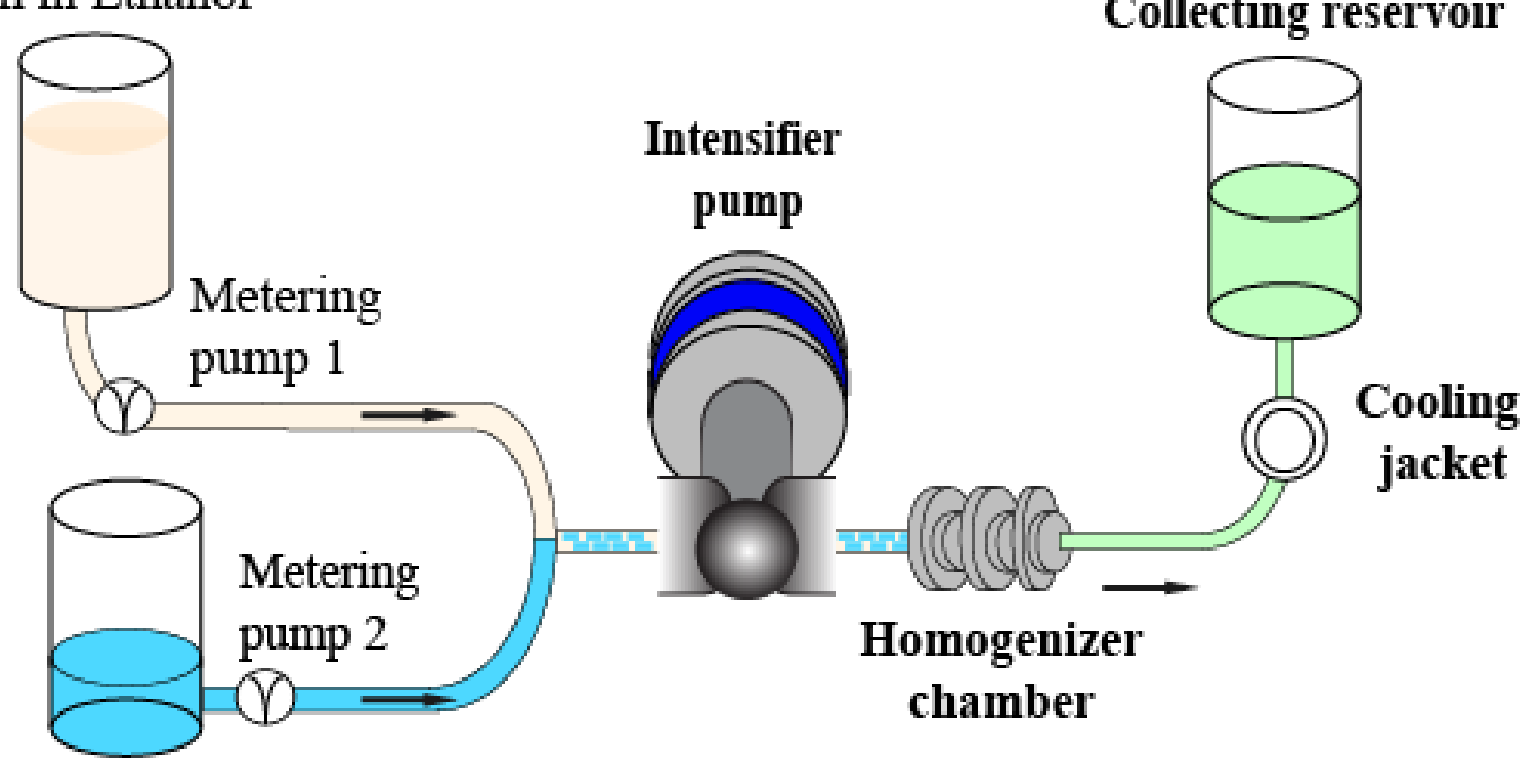

\section{Inlet reservoir 2}

- Caseinate in Water

Figure 1: (a) Schematic illustration of the production of zein nanoparticles using one-step dualchannel microfluidization. A stream of zein in ethanol is made to impinge on a stream of caseinate in water, which results in the formation of zein nanoparticles due to antisolvent precipitation. 


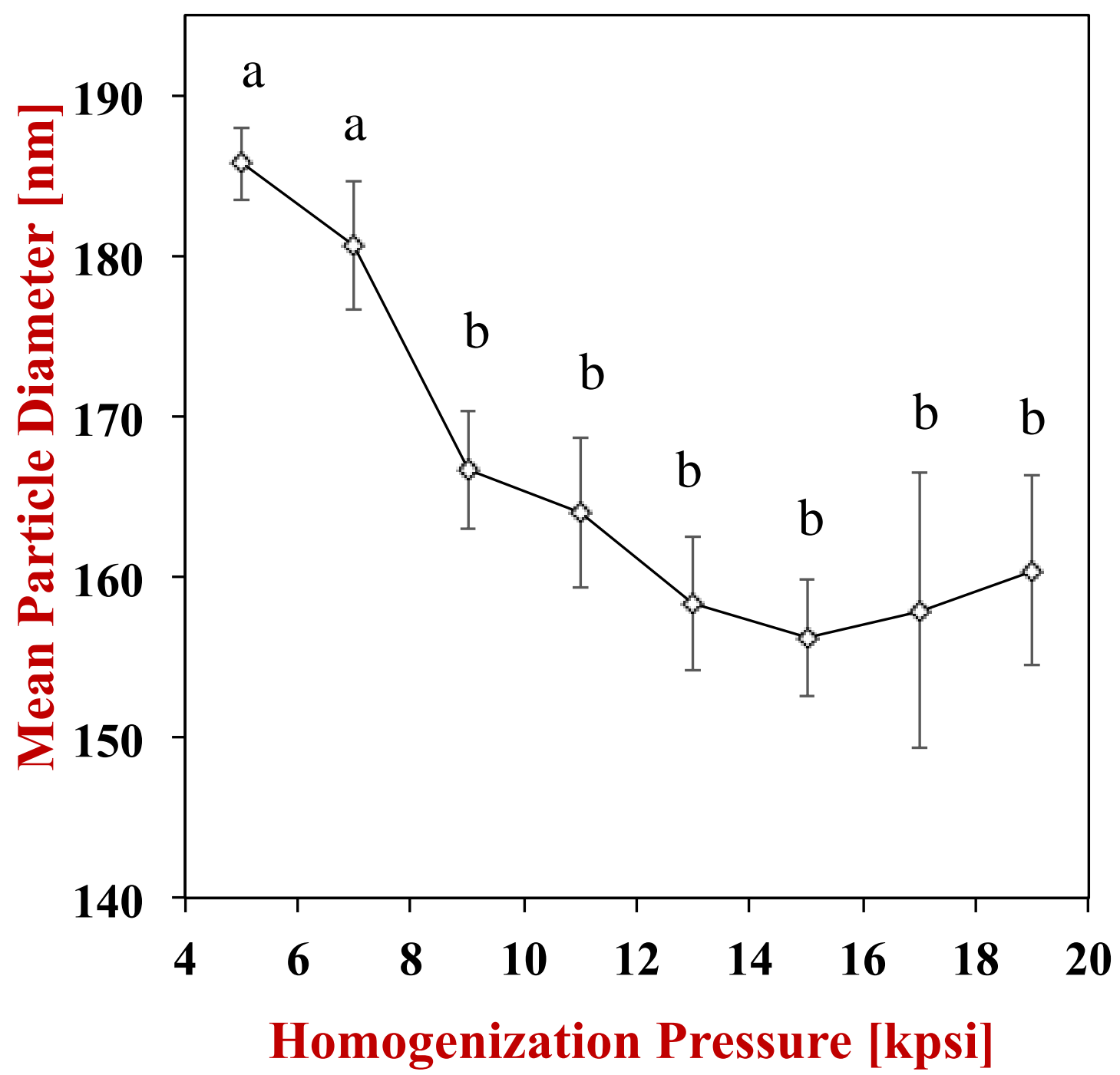

Figure 2a: Influence of homogenization pressure of dual-channel microfluidizer on mean particle diameter of zein nanoparticles produced by antisolvent precipitation (3 \% zein, $80 \% \mathrm{EtOH}$, solvent-to-antisolvent mixing ratio 1:5, 1 \% of sodium caseinate in antisolvent). Different letters above the date points represent statistical difference $(\mathrm{p}<0.05)$. 


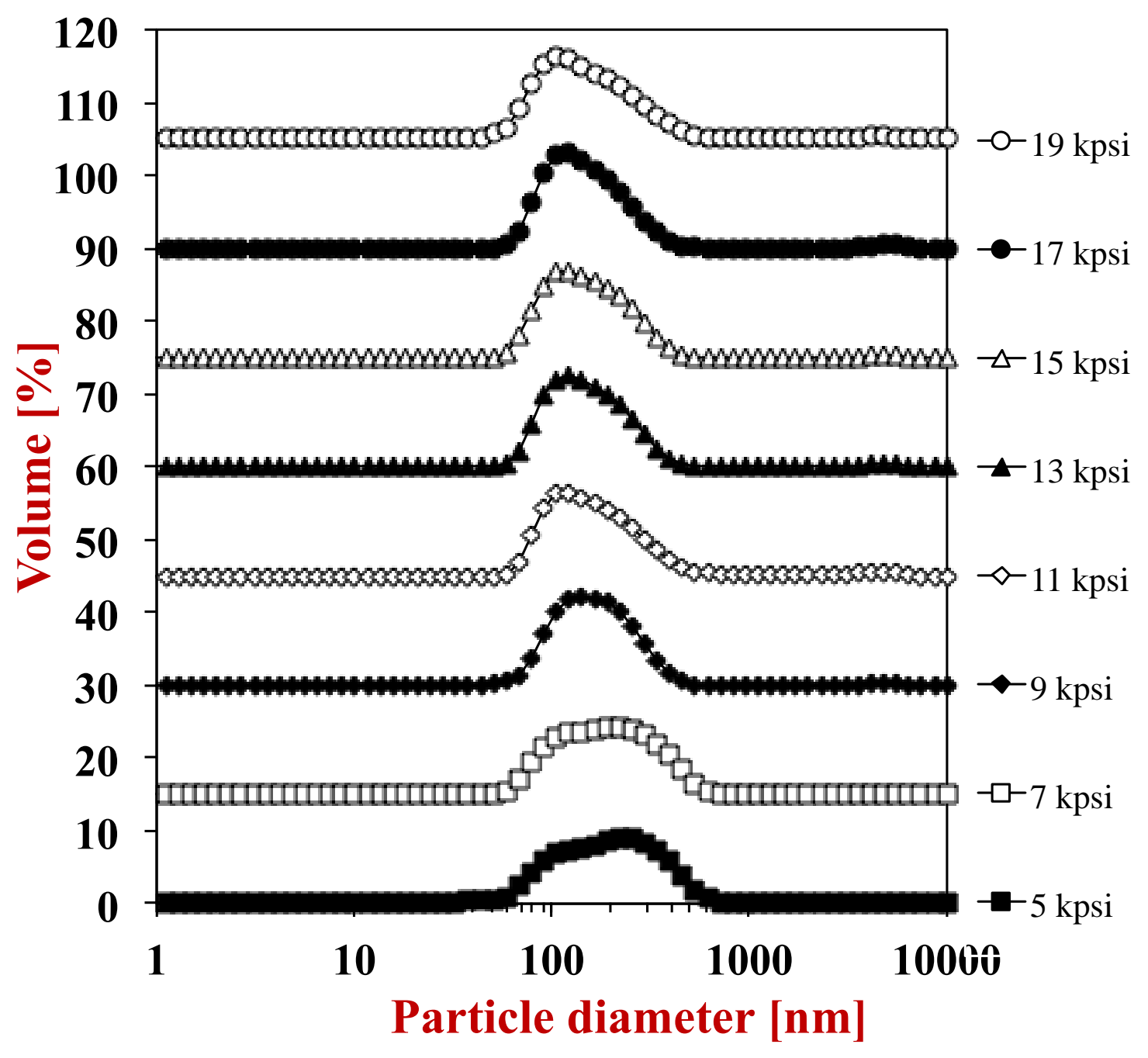

Figure 2b: Influence of homogenization pressure of dual-channel microfluidizer on particle size distribution of zein nanoparticles produced by antisolvent precipitation ( $3 \%$ zein, $80 \% \mathrm{EtOH}$, solvent-to-antisolvent mixing ratio $1: 5,1 \%$ of sodium caseinate in antisolvent). 


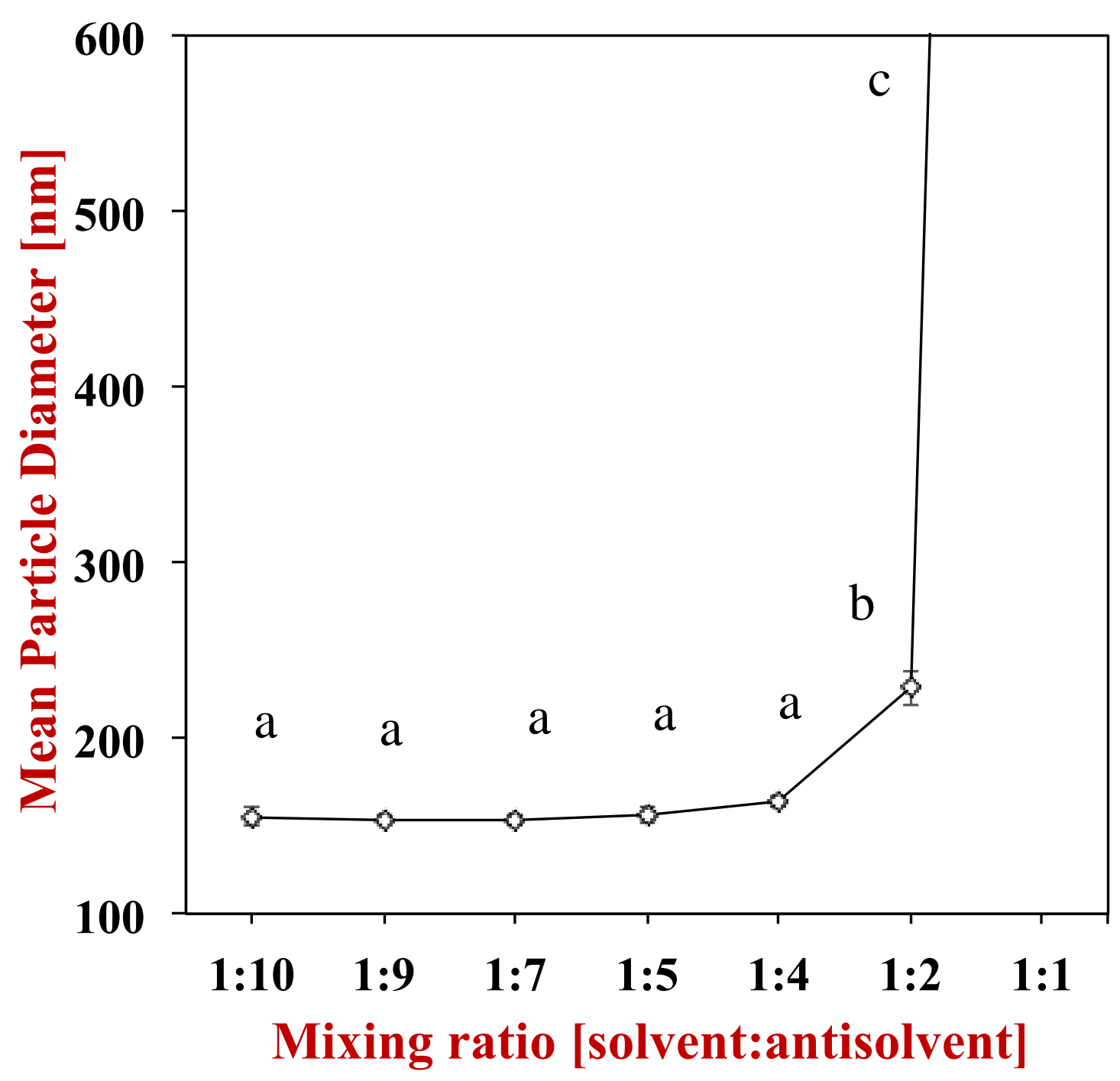

Figure 3a. Influence of mixing ratio of solvent-to-antisolvent on the mean particle diameter of zein nanoparticles ( $3 \%$ zein, $80 \% \mathrm{EtOH}$, pressure $13 \mathrm{kpsi}$ ) produced by dual-channel microfluidization. Different letters above the date points represent statistical difference $(\mathrm{p}<$ $0.05)$. 


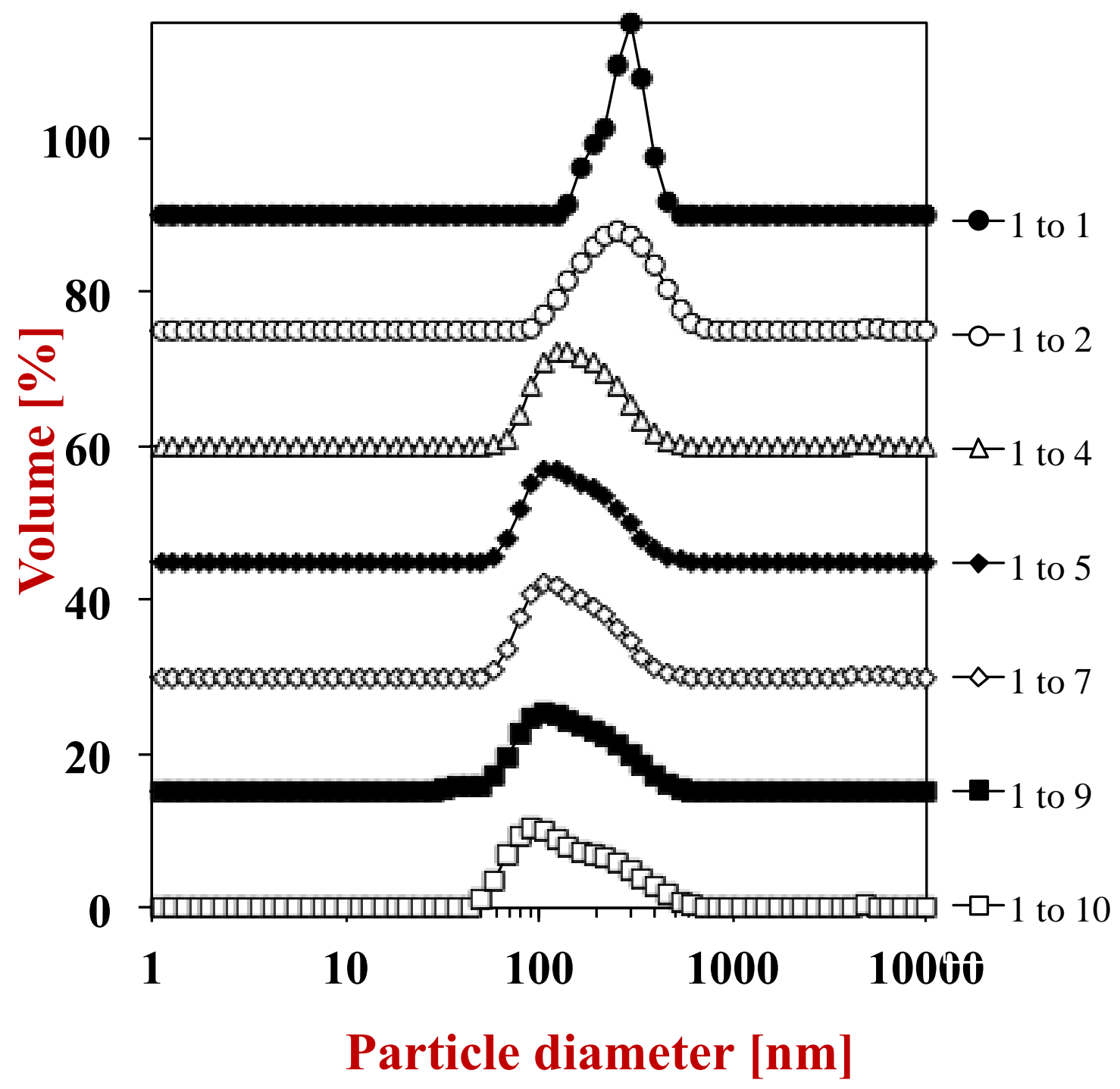

Figure 3b: Influence of mixing ratio of solvent-to-antisolvent on the particle size distribution of zein nanoparticles ( $3 \%$ zein, $80 \% \mathrm{EtOH}$, pressure $13 \mathrm{kpsi}$ ) produced by dual-channel microfluidization. 


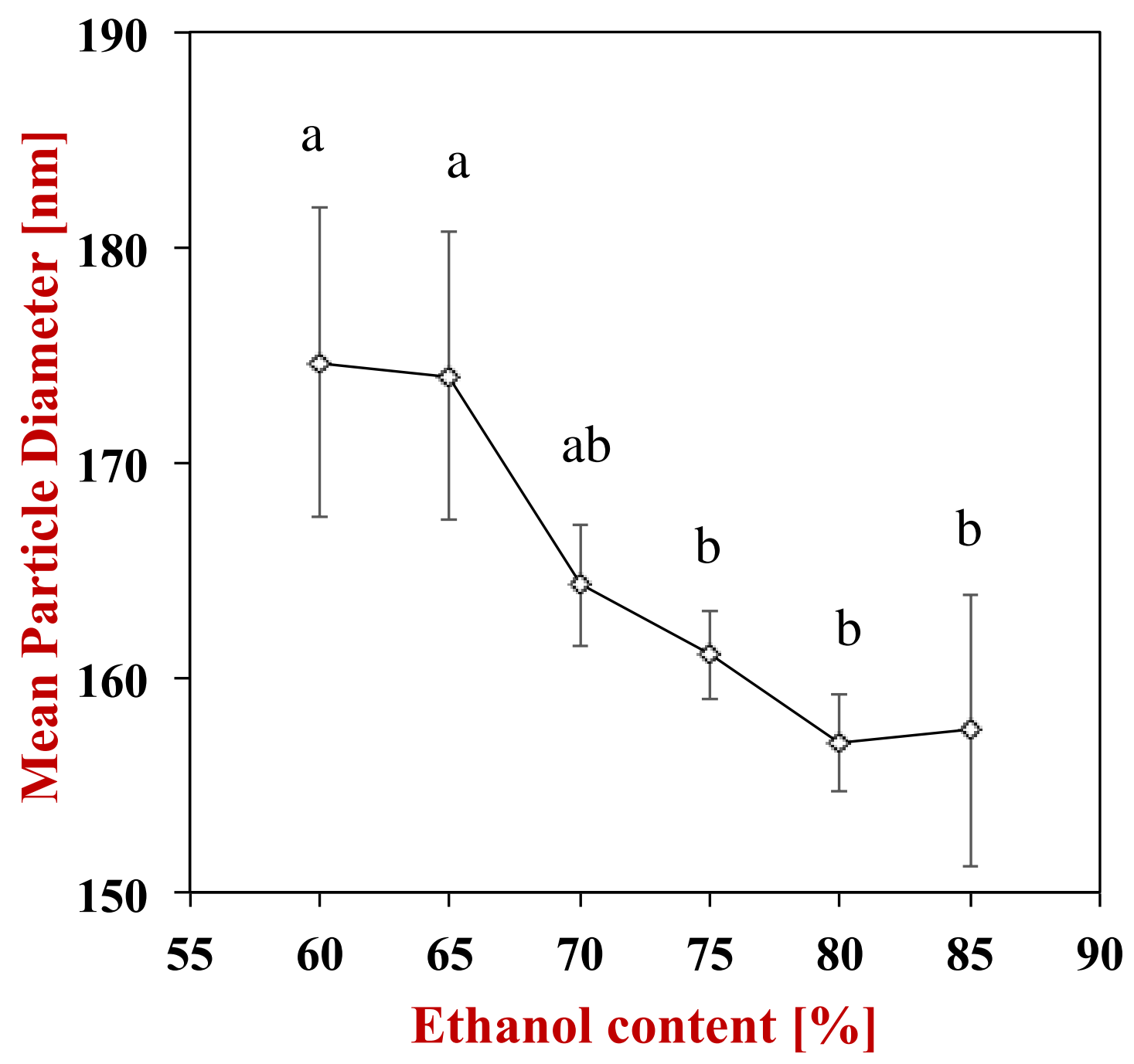

Figure 4a: Influence of ethanol content in the solvent phase on the mean particle diameter of zein nanoparticles produced by dual-channel microfluidization: $3 \%$ zein, mixing ratio 1:5, pressure $13 \mathrm{kpsi}$, and $1 \%$ sodium caseinate in antisolvent. Different letters above the date points represent statistical difference $(\mathrm{p}<0.05)$. 


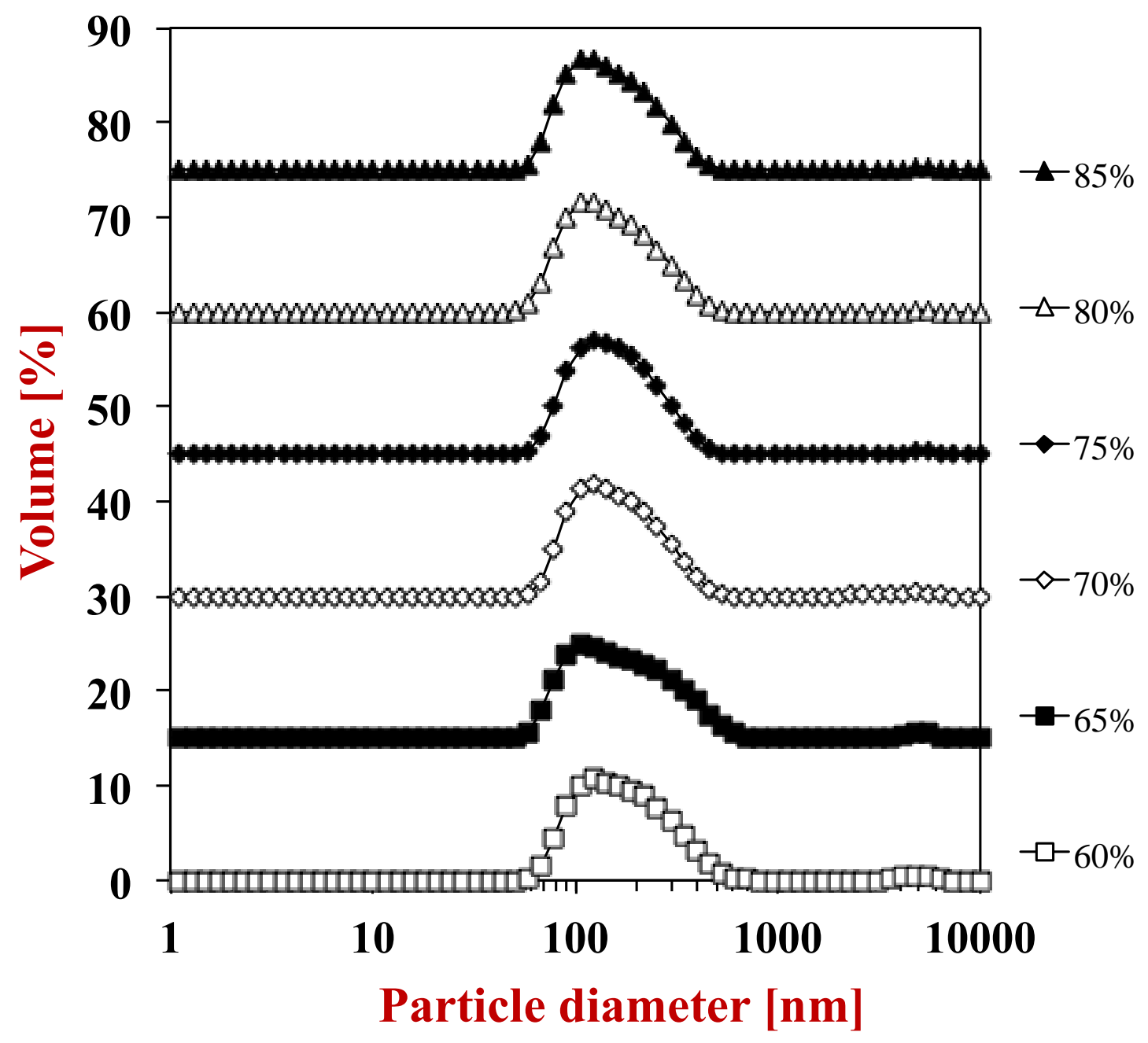

Figure 4b: Influence of ethanol content in the solvent phase on the particle size distribution of zein nanoparticles produced by dual-channel microfluidization: $3 \%$ zein, mixing ratio 1:5, pressure $13 \mathrm{kpsi}$, and $1 \%$ sodium caseinate in antisolvent. 


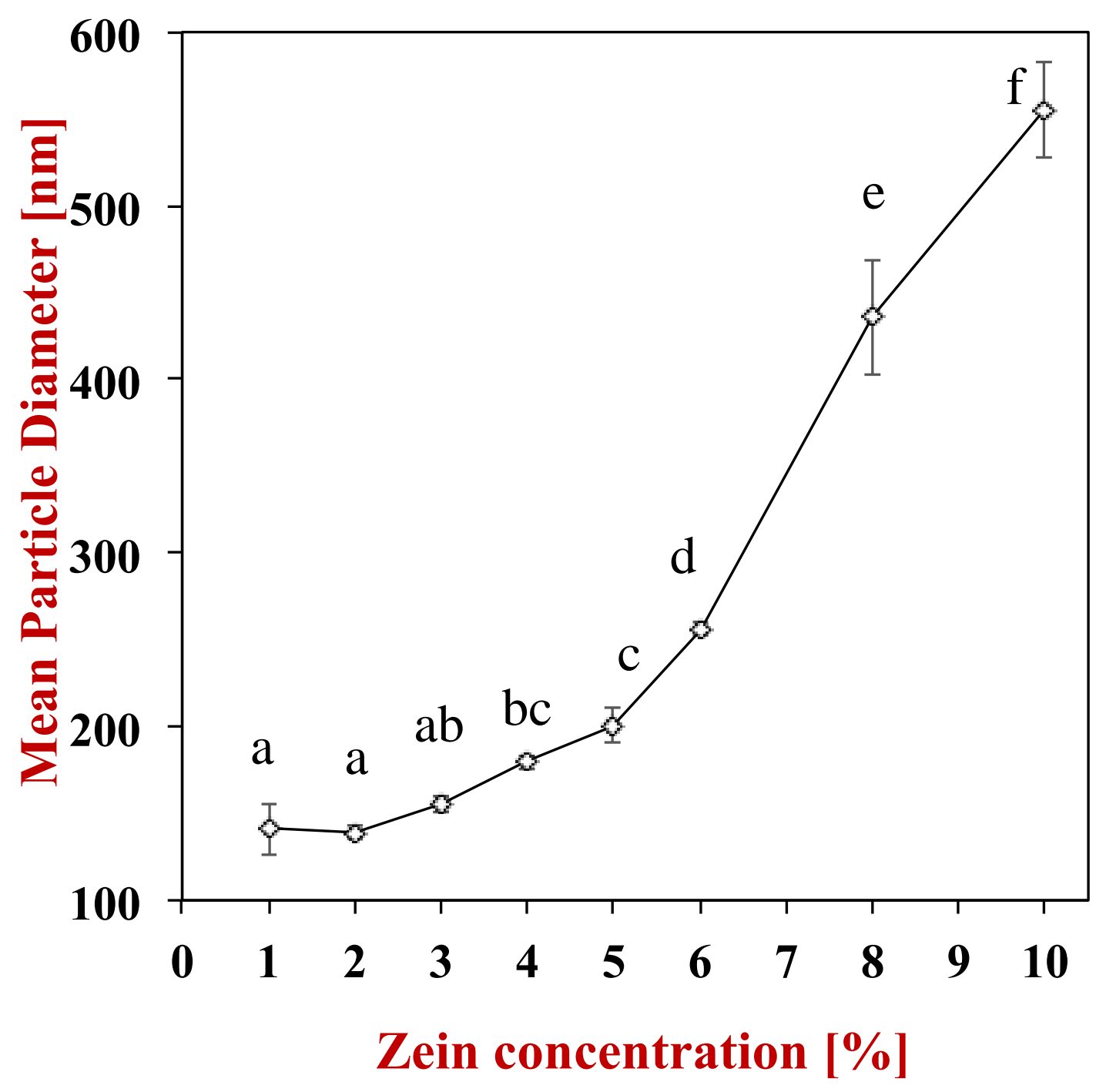

Figure 5a: Influence of zein concentration in the solvent phase on the mean particle diameter of zein nanoparticles produced using a dual-channel microfluidizer: $80 \%$ ethanol (v/v), mixing ratio 1:5, pressure $13 \mathrm{kpsi}, 1 \%$ sodium caseinate in antisolvent. Different letters above the date points represent statistical difference $(\mathrm{p}<0.05)$. 


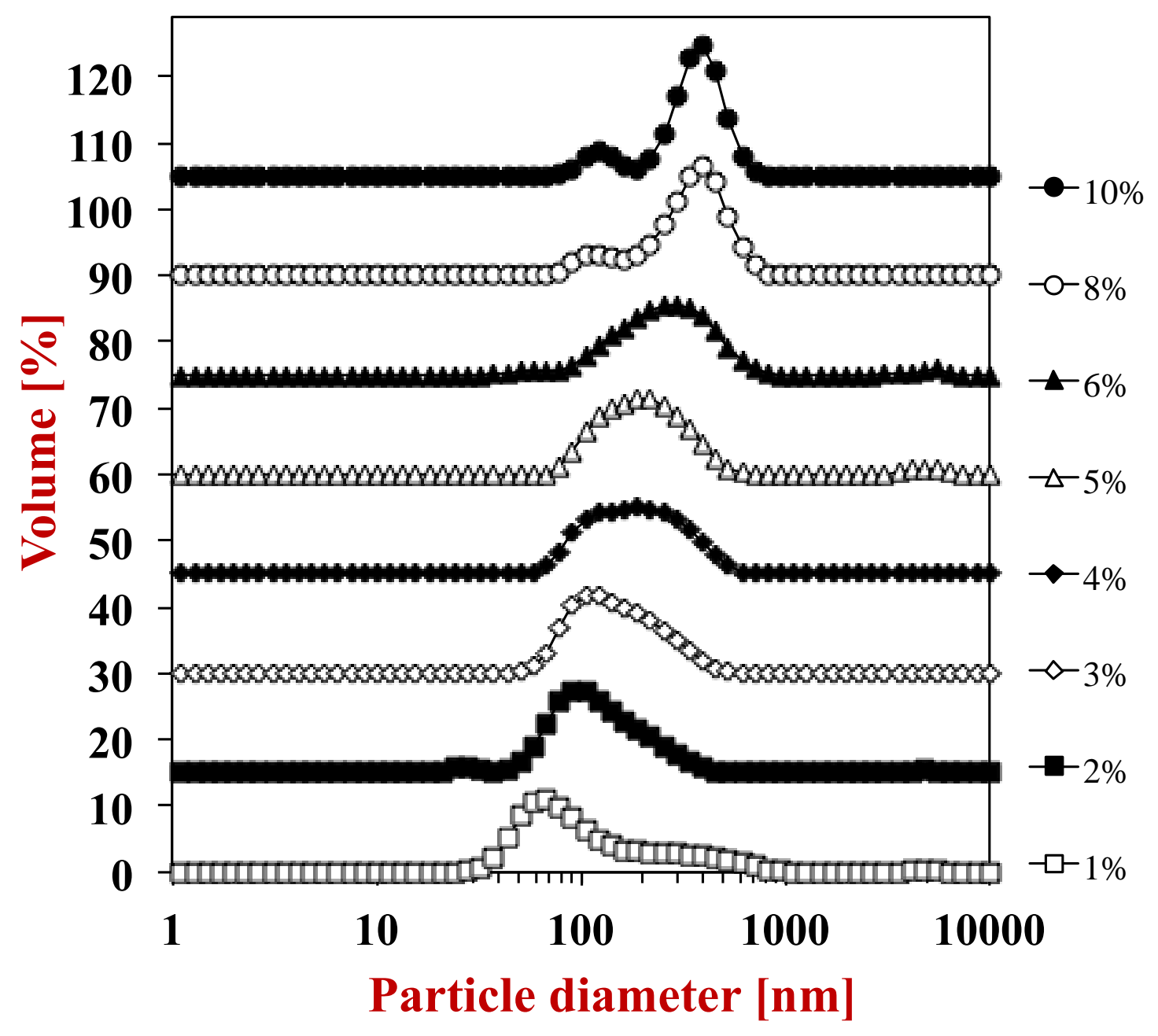

Figure 5b: Influence of zein concentration in the solvent phase on the mean particle diameter of zein nanoparticles produced using a dual-channel microfluidizer: $80 \%$ ethanol (v/v), mixing ratio $1: 5$, pressure $13 \mathrm{kpsi}, 1 \%$ sodium caseinate in antisolvent. 


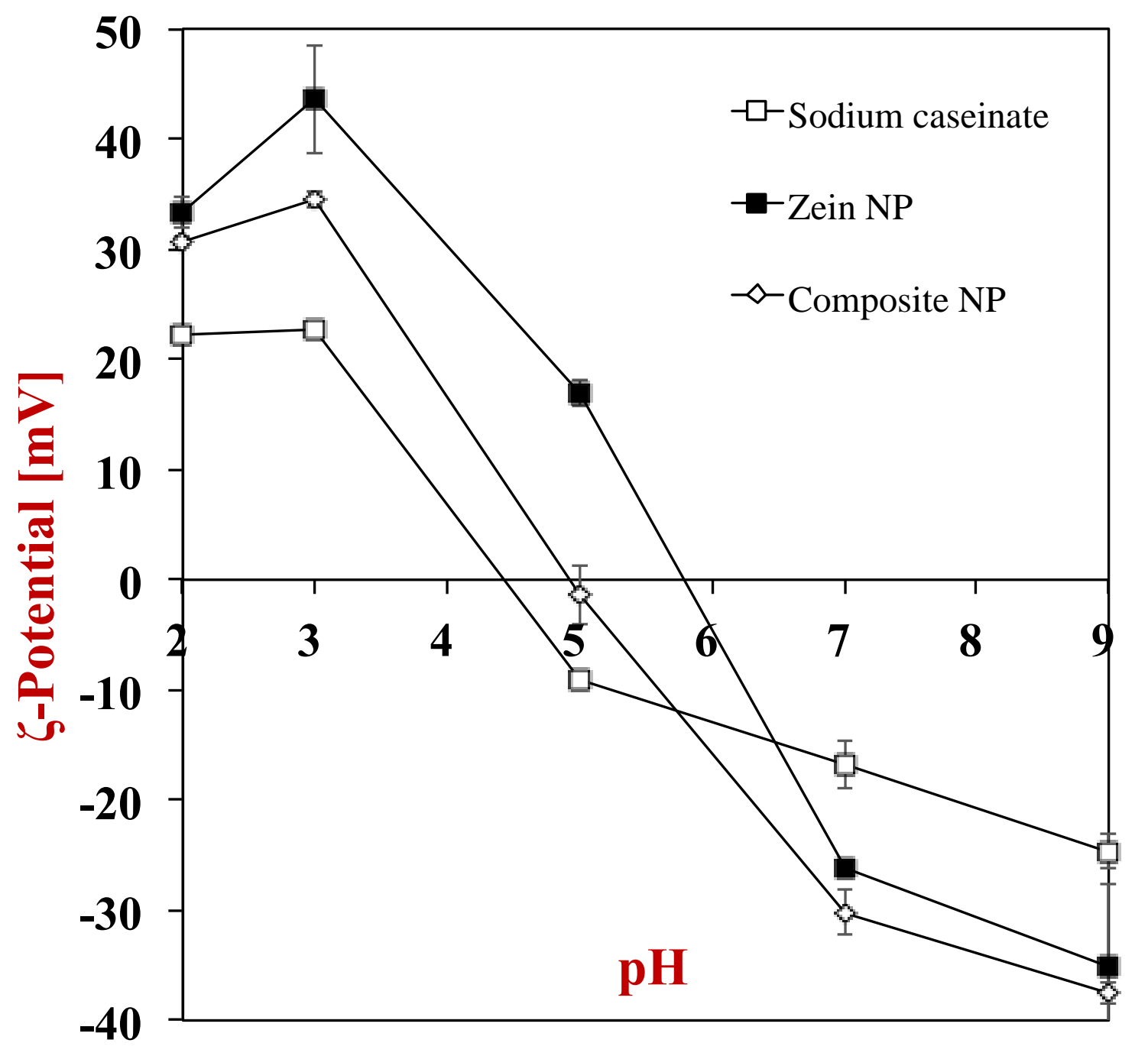

Figure 6a: $\zeta$ potential versus $\mathrm{pH}$ profiles of sodium caseinate solution, bare zein nanoparticles, and composite nanoparticles. The nanoparticles were prepared using optimized conditions (75\% ethanol (v/v), $4 \%$ zein (w/v), $13 \mathrm{kpsi}$, mixing ratio 1-to-5, $1 \%$ of sodium caseinate in antisolvent). 


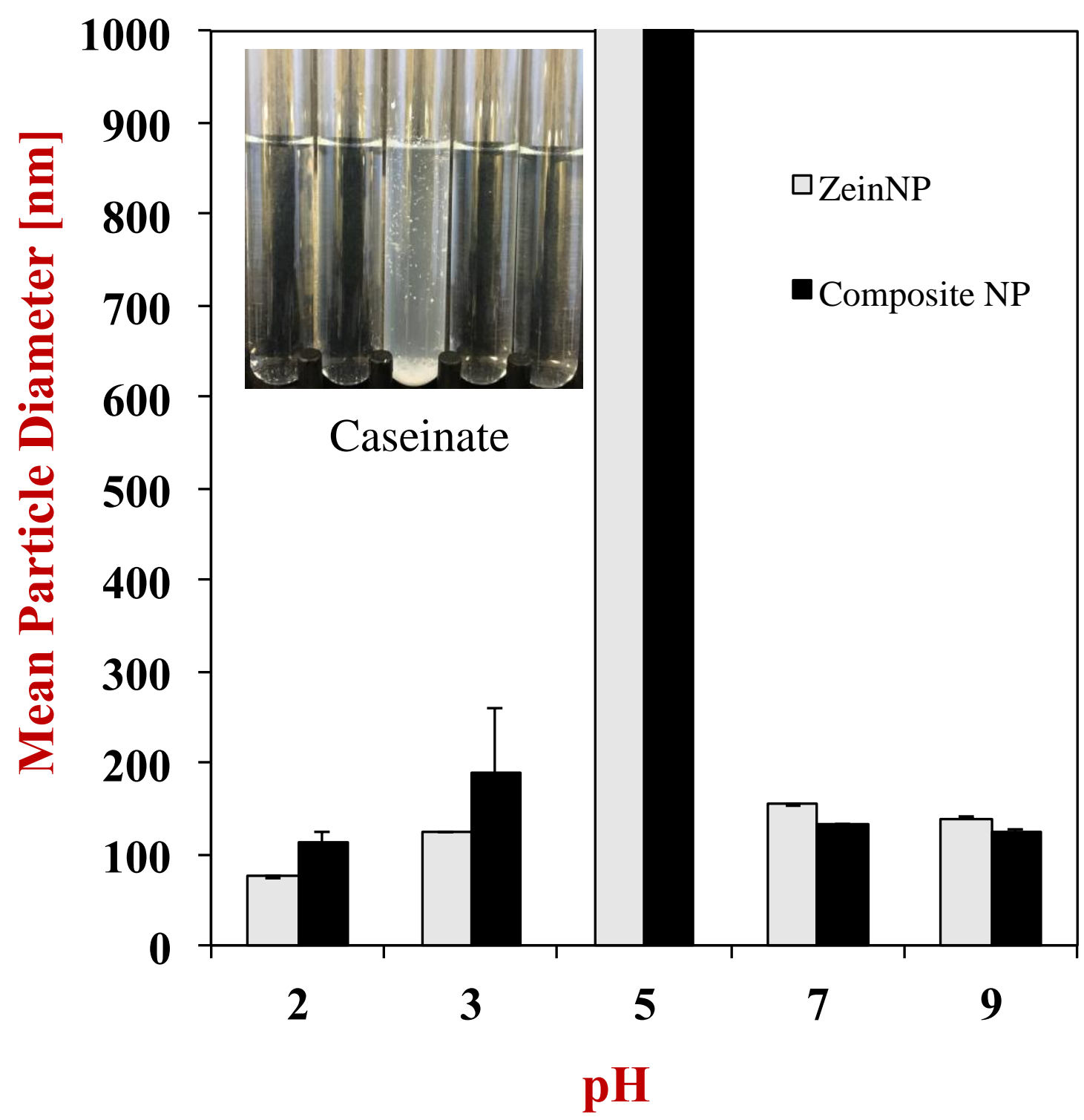

Figure

6b: Particle size versus $\mathrm{pH}$ profiles of bare zein nanoparticles and composite nanoparticles, and impact of $\mathrm{pH}$ on appearance of caseinate solutions. The nanoparticles were prepared using optimized conditions (75\% ethanol (v/v), $4 \%$ zein (w/v), $13 \mathrm{kpsi}$, mixing ratio 1 -to-5, $1 \%$ of sodium caseinate in antisolvent). 


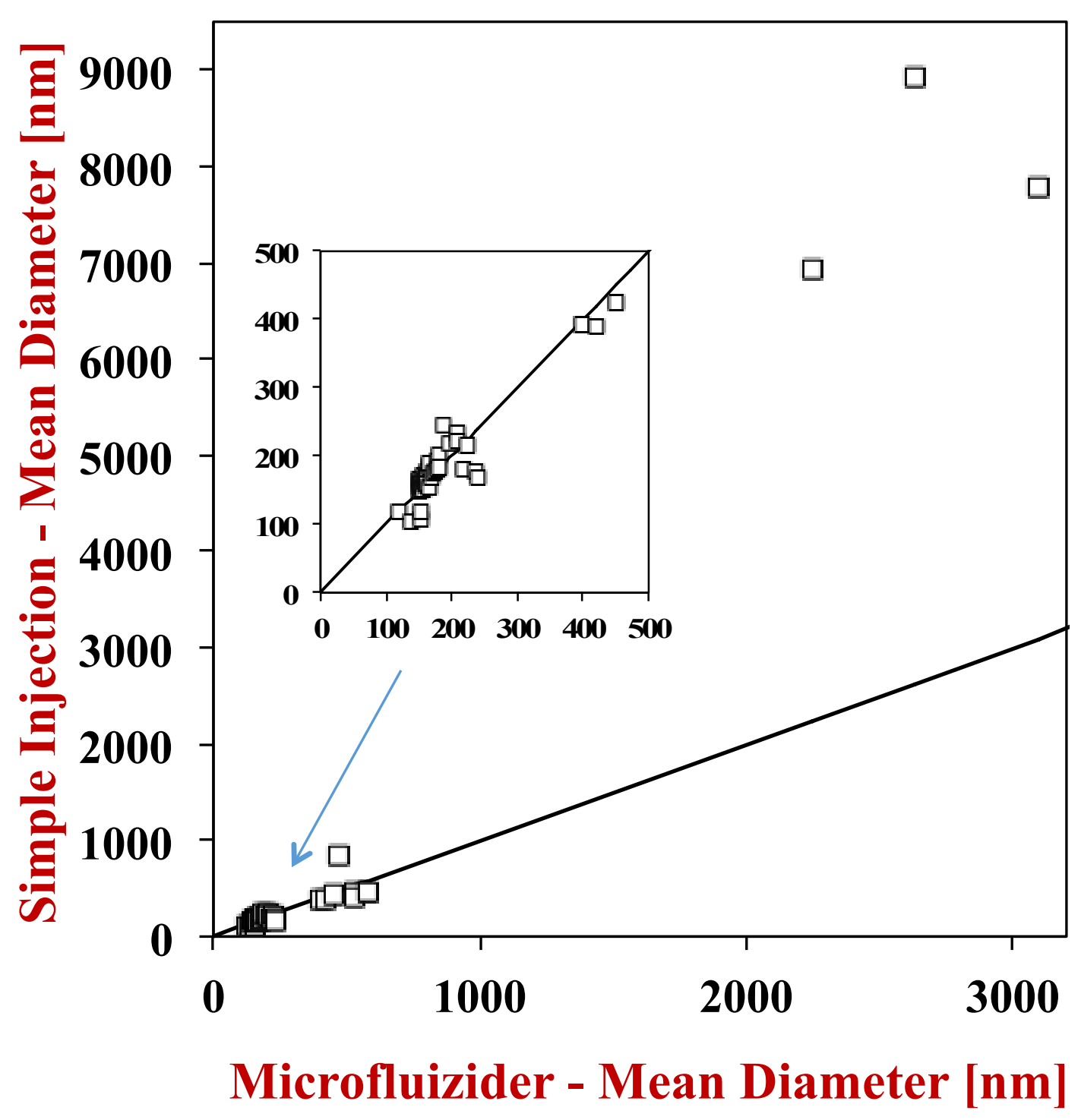

Figure 7: Comparison of the mean particle diameter of zein nanoparticles prepared by simple injection or by microfluidization. The zein concentration in the solvent was $4 \%(\mathrm{w} / \mathrm{v})$, the ethanol content in the solvent was $75 \%(\mathrm{v} / \mathrm{v})$, a mixing ratio of solventto-antisolvent of was 1-to-5, and a sodium caseinate concentration in the antisolvent of $1 \%(\mathrm{w} / \mathrm{v})$ was used to form the nanoparticles. 


\section{Batch approach}
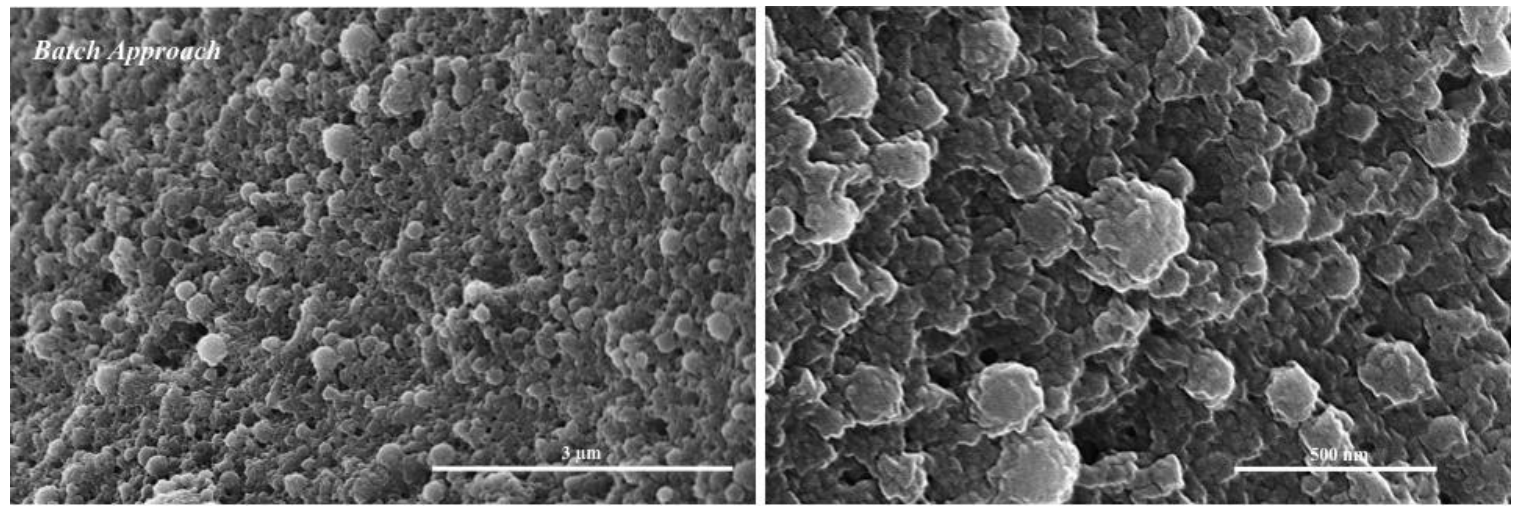

\section{Continuous approach}

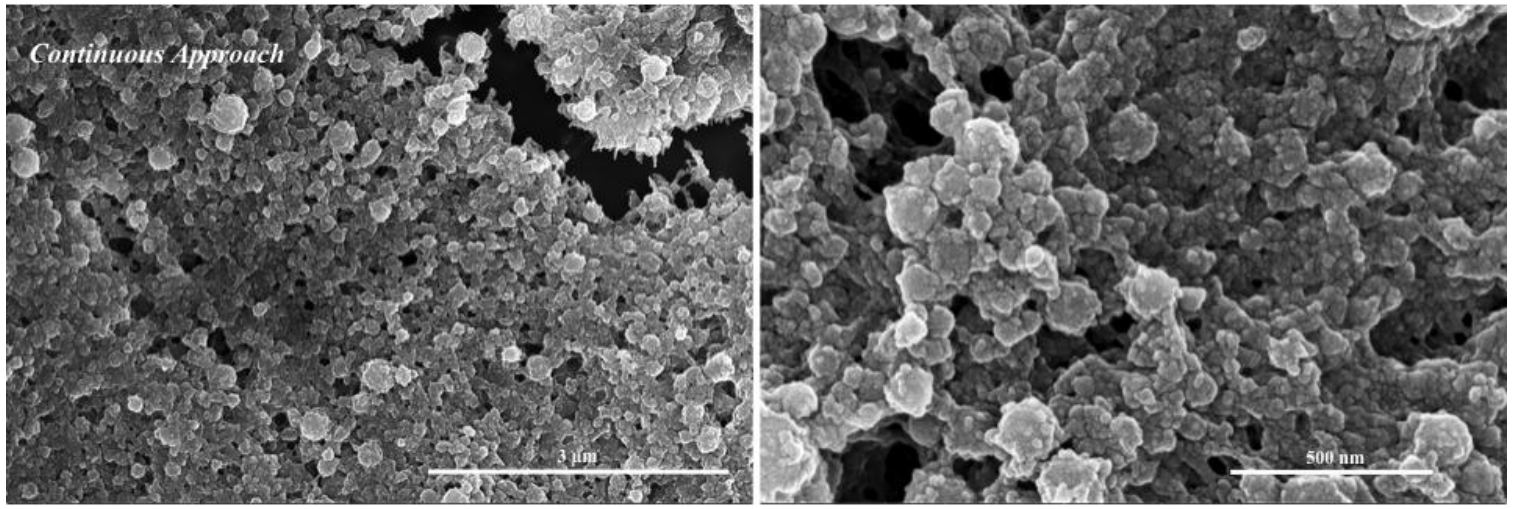

Figure 8: SEM images of caseinate-coated zein nanoparticles at 20k times (left) and 80k times (right) magnification. Antisolvent precipitation was carried out using either the batch or the continuous (microfluidizer) approach and the particle suspensions were freeze-dried prior to SEM analysis. The system consisted of: $4 \%(\mathrm{w} / \mathrm{v})$ zein in solvent; $75 \%$ (v/v) ethanol in solvent; $1 \%(\mathrm{w} / \mathrm{v})$ sodium caseinate concentration in the antisolvent; and, mixing ratio of solvent-toantisolvent $=1-$ to- 5 . 
Inlet reservoir 1

- Zein in Ethanol

\section{Collecting reservoir}

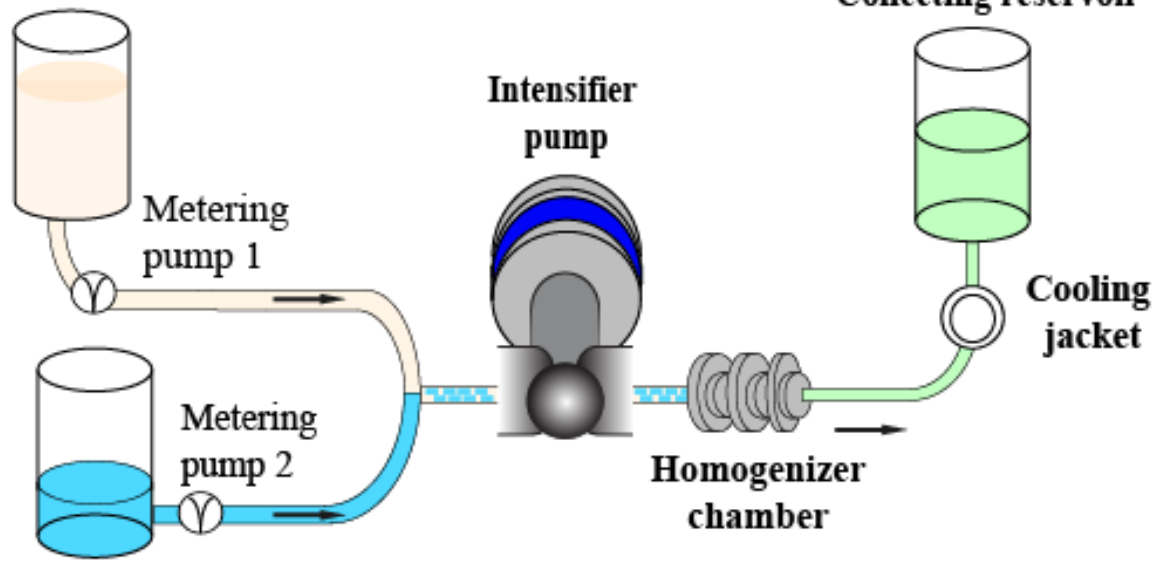

\section{Inlet reservoir 2}

- Caseinate in Water 